\title{
Achim Spiller
}

\section{Meine Lasagne hat mehr PS als dein Auto: Ist die Lebensmittelwirtschaft besonders skandalanfällig?}

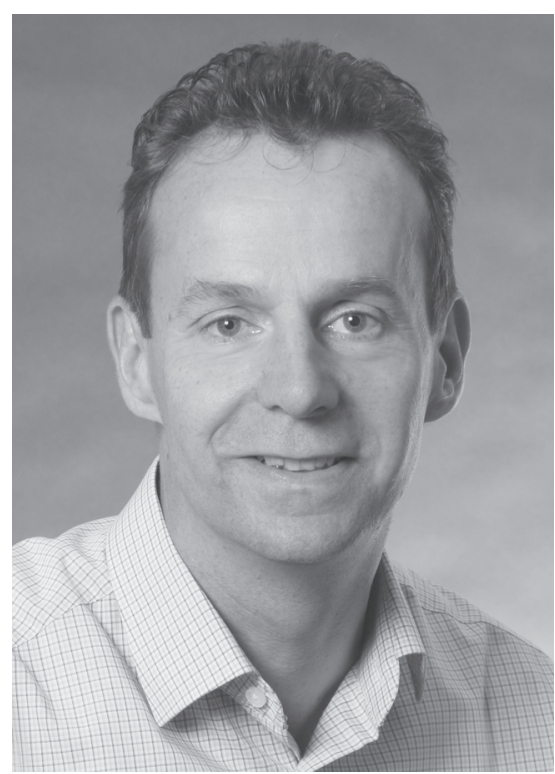

Achim Spiller, Professor für Marketing für Lebensmittel und Agrarprodukte an der Georg-August-Universität Göttingen, O. Mitglied der Göttinger Akademie seit 2013

\section{Pferdefleisch und mehr}

Mein Ruf auf die Professur für „Lebensmittelund Agrarmarketing“ an die Georg-AugustUniversität Göttingen im Jahr 2000 fiel zeitlich mit der BSE-Krise zusammen. Am 24.11.2000 wurde erstmals bei einer Kuh in Deutschland Bovine Spongiforme Enzephalopathie nachgewiesen. Am 9. Januar 2001 traten zwei Minister der Bundesregierung deshalb zurück. Bereits zwei Jahre zuvor war die EU-Kommission u. a. aufgrund der britischen BSE-Fälle vom Parlament zum Rücktritt gedrängt worden. Der BSE-Fall gilt als eine der schwerwiegendsten Politikkrisen der EU. Für mich persönlich bot sie den direkten Einstieg in die Politikberatung durch die Berufung zum Leiter der Arbeitsgruppe Qualitätssicherung in einer vom niedersächsischen Ministerpräsidenten eingesetzten Regierungskommission zur Zukunft der deutschen Agrarwirtschaft. Der folgende Beitrag greift Erfahrungen aus mehr als 10 Jahren

wissenschaftlicher Beschäftigung, Politikberatung (etwa im wiss. Beirat des BMEL für Agrarpolitik) und Wirtschaftsberatung (u. a. als Mitglied und mehrjähriger Vorsitzender des Kuratoriums des weltweit größten Qualitätssicherungssystems QS, aber auch als Initiator einer Tierschutzlabel-Initiative) auf.

Das Thema Lebensmittelskandale hat dabei nichts an Aktualität verloren, in großer Regelmäßigkeit kommt es in den letzten Jahren zu Krisen, Skandalen und gesellschaftlichen Auseinandersetzungen in der Agrar- und Ernährungswirtschaft. Es ist daher keine zufällige Koinzidenz, dass auch meine Berufung in die Akademie der Wissenschaften zu Göttingen und dieser Vorstellungsvortrag von einem Lebensmittelskandal begleitet wurden. Im Frühjahr 2013 hat der Pferdefleischskandal in Europa beachtliche Wellen geschlagen. Die Beimischung preiswerten Pferdefleisches in Rindfleischerzeugnisse hat der Branche zusätzlich einigen Spott eingetragen. Verbraucher sehen sich in ihrem Misstrauen (Kayser et al. 2012a) gegen eine Branche ohne Moral bestätigt. Der folgende Beitrag fragt nach den Ursachen der zahlreichen 
Fleischkrisen. In meinen Forschungsarbeiten habe ich dabei zunächst an den direkten akteursbezogenen Erklärungen angesetzt (Anreize zu opportunistischem Verhalten, Kontrolldefizite), in den letzten Jahren dann, zunehmend interdisziplinär, kulturelle Hintergrundfaktoren in den Blick genommen.

\section{Opportunistisches Verhalten, Kontrolllücken und die Ökonomie des Verbrechens}

Diskussionen über die Ursachen der hohen Krisendichte in der Fleischwirtschaft setzen auf unterschiedlichen Ebenen an. Akteursbezogen ist die Skandalintensität Ergebnis einer kleinteiligen Branche unter starkem Preisdruck. Die Fleischindustrie ist zudem weitgehend anonym im Sinne „namenloser“ Anbieter, die der breiten Öffentlichkeit unbekannt sind. Markenartikel sind kaum verbreitet (Schramm et al. 2004). Ökonomisch liegt hier eine Situation mit hohen Betrugsanreizen, geringen Aufdeckungswahrscheinlichkeiten und niedrigen Sanktionsdrohungen vor. Es ist daher für Unternehmen nicht selten rational, die Betrugsvariante zu wählen - etwa wenn beim Pferdefleischskandal Pferdefleisch für weniger als 0,5 €/kg gehandelt wird. Geschmacklich bei Beimischung kaum zu identifizieren, war die Aufdeckungswahrscheinlichkeit eines Betrugs eher gering (Europäisches Parlament 2013). Die betrügenden Fleischgroßhändler gehörten vornehmlich in die Kategorie der sog. „Fly by Night“-Anbieter, die keinen Namen zu verlieren hatten und nach Aufdeckung unter anderen Namen weiter am Markt auftreten konnten.

Die ökonomische Theorie des Verbrechens schreibt Kontrollsystemen und Strafdrohungen eine besondere Bedeutung zu, da strikte Überwachung und hohe potenzielle Sanktionen Betrug in der Abwägung der Unternehmen unattraktiv werden lassen. Bis zur BSE-Krise lag die Verantwortung dabei fast ausschließlich auf den Schultern der staatlichen Lebensmittelüberwachung. Diese war historisch eine Reaktion auf Fleischskandale und Zustände in den ersten Formen industrieller Lebensmittelproduktion, den Schlachthäusern in Chicago, wie sie Upton Sinclair in The Jungle (1906) berühmt gemacht hat. Vor diesem Hintergrund hat der Staat in Deutschland Ende des 19. Jahrhunderts/Anfang des 20. Jahrhundert ein System kommunaler Fleischkontrolle eingeführt („Fleischbeschau“), das in seinen Grundzügen bis heute die Überwachung prägt. Staatliche Kontrollen durch einen amtlichen Veterinär am Schlachtband wirken in einer international vernetzten Branche heute allerdings arg anachronistisch (Schulze et al. 2008a). Die Organisation des gesundheitlichen Verbraucherschutzes in Deutschland auf Stadt- oder Landkreisebene ist immer noch durch nicht abgestimmte Analysemethoden und fehlende Datenschnittstellen charakterisiert. Bei der Ehec-Krise des Jahres 2011, die wahrscheinlich durch mikrobakteriell verunreinigte Sprossen aus Ägypten ausgelöst wurde, musste das nach der BSE-Krise neu gegründete Bundesinstitut für Risikoforschung durch einige Dutzend Mitarbeiter händisch Warenlieferscheine und Analysedaten in ein EDV-System übertragen lassen, um Transparenz über die zugrunde liegenden Warenströme zu gewinnen. 
In meinen Arbeiten habe ich mich vor diesem Hintergrund intensiv mit dem Aufbau privatwirtschaftlicher Systeme der Lebensmittelkontrolle beschäftigt (Theuvsen et al. 2007). Auf Basis entsprechender Konzepte aus der Luftfahrt- und Automobilindustrie wurden nach der BSE-Krise kooperative Qualitätssicherungssysteme etabliert, die entlang der gesamten Wertschöpfungskette der Fleischwirtschaft von der Herstellung der Futtermittel über die Landwirtschaft bis zum Lebensmittelhandel Überwachungen vornehmen. Die beiden bedeutendsten Varianten sind das QS-System mit dem Schwerpunkt Fleisch und Globalgap mit dem Fokus auf Obst und Gemüse. Erhebliche Verbreitung hat auch das halbstaatliche Ökokontrollsystem zur Überwachung von Bio-Lebensmitteln. In jüngerer Zeit entstehen weitere Systeme z. B. für traditionelle Lebensmittelspezialitäten, Tierschutz oder nachhaltigen Fischfang.

In diesen Systemen werden private Kontrolleure (Zertifizierungsunternehmen) von den zu überwachenden Unternehmen auf Basis eines zugrunde liegenden Qualitätsstandards mit der Kontrolle beauftragt. Im Erfolgsfall wird ein Zertifikat ausgestellt, das den Kunden des Unternehmens Sicherheit beim Wareneinkauf bieten soll. Das basale Spannungsverhältnis wird sofort offenbar, wenn der zu Kontrollierende seinen Kontrolleur bezahlt. Der Zertifizierer hat ein Interesse, seinen Kunden nicht durch eine zu strenge Kontrolle zu verärgern, dies um so mehr, wenn Folgeaufträge lukrativer als Erstaufträge sind, da man in diesem Fall den Kunden schon besser kennt und schneller prüfen kann. Diese prekäre Anreizsituation ist evident und Ökonomen aus der Wirtschaftsprüfung bekannt. Wir haben daher in Forschungsarbeiten auf Basis von Modellen der Wirtschaftsprüfungstheorie untersucht, wie der das Gesamtsystem überwachende Systemträger (Verbände oder Staat) durch seine Kontrolle der Kontrolleure die Effizienz des Gesamtsystems sicherstellen kann (Jahn et al. 2005). Auf Basis empirischer Studien konnten wir z. B. nachweisen, wie unterschiedlich solide verschiedene Zertifizierer gearbeitet haben und Vorschläge zur Verbesserung der Kontrollpraxis vorschlagen (Albersmeier et al. 2009). So wurden z. B. die vormals angekündigten Kontrollen des QS-Systems in den letzten Jahren zunehmend durch unangekündigte, risikoorientierte Stichprobenkontrollen ergänzt. Dies erhöht die Aufdeckungswahrscheinlichkeit für potenzielle Betrüger beachtlich (Padilla Bravo et al. 2013).

\section{Macho-Kultur der harten Männer: Fleischskandale und Branchenkultur}

Trotz des Ausbaus der Qualitätssicherung haben internationale Fleischgroßhändler über Jahre hinweg erfolgreich Pferdefleisch in die Branche verkauft - teilweise mit Wissen der Einkäufer, die daraus Wurst und Fertiggerichte wie die titelgebende Lasagne gefertigt haben. In der Branche hat sich durch den Umgang mit Tier und Tod eine „Macho-Kultur der harten Männer“(Spiller 2009) herausgebildet, die opportunistisches Verhalten begünstigt. Die Fleischwirtschaft ist nicht nur die größte Branche der deutschen Ernährungsindustrie, sie verfügt auch über eine ausgeprägte Bran- 
chenkultur: Vielfach werden Geschichten über das aggressive Arbeitsklima kolportiert, etwa über Führungskräfte, die beim Betriebsrundgang den Arbeitern besser „nicht den Rücken zukehren sollten“. Mordfälle in der Branche haben mehrfach die Kulisse für Tatort-Filme abgegeben - nicht ganz ohne realen Hintergrund, wie die rechtskräftige Verurteilung eines Viehhändlers für die Beauftragung eines Mordanschlags gegen seinen wichtigsten Konkurrenten im Jahr 2012 andeutet.

Die Fleischwirtschaft ist eine typische Selfmade-Branche. Es gibt einen geringen Akademisierungsgrad. Sie ist stark regional konzentriert. Besonders hervorstechend ist die Weser-Ems-Region, ursprünglich ein landwirtschaftlich benachteiligtes Gebiet mit schlechten Böden und kleineren Betrieben. Inzwischen wird in der Region jedes zweite deutsche Hähnchen und jedes dritte Schwein gemästet. Es gibt in dieser Region eine starke Vernetzung über die Wertschöpfungsstufen und einen ausgeprägten Regionalstolz, kombiniert mit einem dicht gewebten formellen und informellen Netzwerk zwischen Wirtschaft und Politik. Diese Abgeschlossenheit verstärkt die branchenkulturelle Homogenität der Kernunternehmen in der deutschen Fleischwirtschaft.

Auf Basis der aus der internationalen Kulturforschung stammenden Kriterien von Hofstede (1980) lässt sich die Branchenkultur der Fleischwirtschaft folgendermaßen darstellen. (1) Uncertainty Avoidance: Die Regelbindung des Managementhandelns ist eher schwach ausgeprägt, die Risikobereitschaft hoch. Es dominiert kurzfristiges, gelegenheitsgetriebenes Entscheiden. Die Bereitschaft, auf Spot-Märkten in der Fleischwirtschaft riskante Geschäfte zu tätigen, ist groß. (2) Masculinity: Frauen in Führungspositionen finden sich in der Branche nur im Ausnahmefall. Es herrschen eher rüde Umgangsformen, wie sie für stark maskulin geprägte Gruppen typisch sind. Klassische Rollenmodelle und eine kulturelle Besetzung des Fleischkonsums als männliches Produkt sind verbreitet. (3) Power Distance: Die Branche ist durch relativ flache, aber ausgeprägte Hierarchien und einen autokratischen Führungsstil geprägt. Machtdistanzen werden toleriert, Gegenmachtkonzepte (z. B. Betriebsräte) erschwert. (4) Individualism: Die Fleischwirtschaft ist durch starke Unternehmerpersönlichkeiten geprägt. Erfolg wird eher einzelnen Entscheidern als einer Betriebsgemeinschaft zugesprochen. Die Fleischwirtschaft ist damit im Sinne von Deal/Kennedy (1982) eine idealtypische „Tough-guy macho culture“ mit schnellem Feedback und hohem Risiko bei ausgeprägter Kurzfristorientierung.

Eine Facette der Branchenkultur, die für die Probleme der Fleischwirtschaft besonders charakteristisch ist, bildet die geringe Öffentlichkeitsorientierung (Albersmeier et al. 2008). Unternehmen können grundsätzlich in vier verschiedene Netzwerke eingebunden sein (Abrahamson/Fombrun 1992):

1. Das Netzwerk der Unternehmen, das in einer Branche über Verbände und geschäftliche Kontakte konstituiert wird.

2. Staatliche bzw. politische Netzwerke, die im europäischen Mehrebenenmodell vielfache vertikale und horizontale Bezüge aufweisen. 


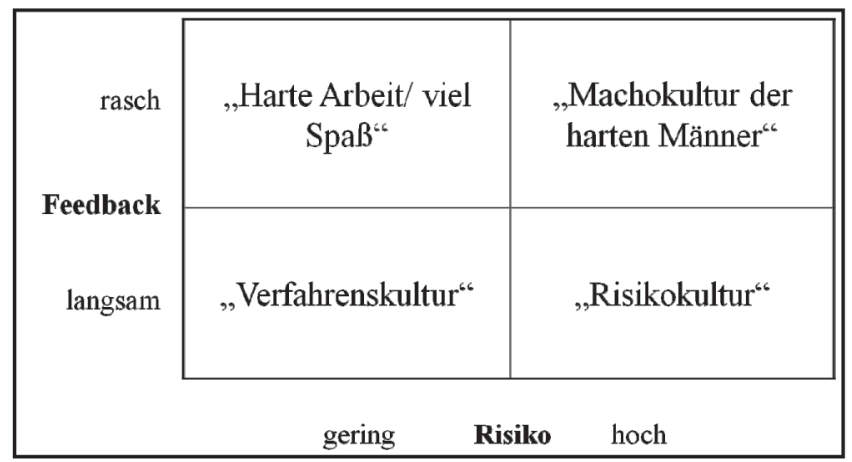

Abb. 1. Unternehmenskulturtypologie nach Deal/Kennedy. Quelle: Deal/Kennedy (1982).

3. Zivilgesellschaftliche Netzwerke, d.h. Kontakte zu NGOs, Kirchen, Vereinen und engagierten Einzelpersonen.

4. Netzwerke der Massenmedien und Kulturinstitutionen, die die moderne Aufmerksamkeitsökonomie prägen und Themen setzen.

Die deutsche Fleischwirtschaft ist intern eng vernetzt und weist gute Drähte zur Agrarpolitik auf, während die Verknüpfungen zur Zivilgesellschaft dünn sind. Die Produktionszentren der Massenmedien konzentrieren sich immer stärker auf urbane Metropolen, entsprechend gering ist der Kontakt der ländlichen Branche zu den kulturell-kreativen Milieus der Gesellschaft (Florida 2002). Die Darstellung der Fleischproduktion in den Medien ist, wie wir in einer Presse- und Internetanalyse zeigen konnten, ausgesprochen negativ (Kayser et al. 2012a). Auf diese Kritik reagiert die Branche mit Unverständnis und Abkopplung, einem Rückzug in die „Wagenburg ihrer regionalen Cluster“. In einer Studie haben wir die Öffentlichkeitsarbeit der Fleischwirtschaft am Beispiel der sog. Gammelfleischskandale mittels einer quantitativen Medienanalyse untersucht (Schulze et al. 2008a). Die großen Unternehmen der Branche sind ebenso wie der Verband der Fleischwirtschaft fast nur in der Fachöffentlichkeit präsent. Sie überlassen den Mediendiskurs der Politik, Journalisten und NGOs. Kontakte zur Zivilgesellschaft sind schwach: Mit einer Befragung von PR-Verantwortlichen der gesamten Agrar- und Ernährungswirtschaft konnten wir zeigen, dass kritische Stakeholdergruppen in der Einschätzung der Industrie die geringste Bedeutung unter allen Zielgruppen der Öffentlichkeitsarbeit haben (Albersmeier et al. 2008).

Die Auseinandersetzungen zwischen der Branche und der Gesellschaft sind kulturell verankert. Der Konflikt spitzt sich dabei durch den beachtlichen wirtschaftlichen Erfolg der Unternehmen in den letzten Jahren zu. Eine starke Branchenkultur kann durch die Abschottung gegenüber rivalisierenden kulturellen Deutungen zu Friktionen und zu einer Einengung des Entscheidungsfeldes führen. Als Beispiele 
können der Umgang der Fleischwirtschaft mit den Themen Bioproduktion und Tierschutz dienen. Konsumentenstudien zeigen, dass Fleisch die Warengruppe mit der höchsten Verbraucherverunsicherung ist (Schulze/Spiller 2008). Gleichwohl ist der Bio-Anteil hier mit unter einem Prozent Marktanteil außergewöhnlich niedrig. Statt die Marktchancen von Premiumsegmenten zu nutzen, verstrickt sich die Branche in Abwehrdiskurse - vielleicht auch, weil die Bio-Branche als feminin geprägte, weniger hierarchische und eher kollektivistische Gruppe einen Gegenpol zur Fleischwirtschaft bildet. Ähnlich wie im Bio-Markt agiert die Branche auch bei der Tierschutzfrage. Unsere Befragungen von Landwirten zeigen die Dominanz eines biological functioning-Paradigmas, einem Frame, in dem hohe biologische Leistungen eines Tieres (Tageszunahmen, Milchleistung etc.) automatisch mit Tierwohl gleichgesetzt werden (Franz et al. 2012). Vor diesem Hintergrund wird Tierschutz häufig als Angriff auf das Berufsethos wahrgenommen.

\section{Agrarromantik und Massentierhaltung}

Die Diskussionen um Tierschutz und Bio-Lebensmittel zeigen, dass Fleischkrisen nicht nur das Ergebnis betrügerischen Verhaltens, sondern Bestandteil eines übergreifenden landwirtschaftlichen Diskurses um Produktionsformen sind. In den letzten Jahren haben wir diese Auseinandersetzungen um Produktivität versus Natürlichkeit in den Fokus der Forschungsarbeiten gerückt (Böhm et al. 2010, Rojas-Méndez et al. 2012, Kayser et al. 2012a). Zum Leidwesen vieler Branchenvertreter ist die Landwirtschaft keine „ganz normale Branche“. Moderne Produktionsverfahren werden zumeist nicht als technischer Fortschritt, sondern als kostenreduzierende, aber qualitätsverschlechternde Entwicklungen wahrgenommen. In der Bevölkerung dominiert der Eindruck, dass Lebensmittel eigentlich zu billig“ sind (Schulze et al. 2008b). Dieses „eigentlich“ drückt die ambivalente Position zu Lebensmittelpreisen aus: Die Diskrepanz zwischen einem Discountanteil von rund 50\% des Marktes und der gleichzeitigen Kritik an nicht kostendeckenden Preisen, die Landwirten keine naturnahe Produktion ermöglichen, wird als Consumer-Citizen-Gap zunehmend thematisiert.

Der Vorwurf aus der Branche lautet dagegen „Agrarromantik im Heidiland“ (Fischler 2012). Science versus Romance betitelt ein Meinungsforschungsinstitut seine Studie über Verbraucheransprüche an Ernährung (Hauser 2012). Als Food-Wars bezeichnen Lang/Heasman (2004) in einem viel beachteten Buch den wachsenden Konflikt zwischen dem produktivitätsorientierten Mainstream der Branche und den Akteuren, die sich mit Bio, Regio, Animal Welfare und Genuss in den Nischen gegen die dominierende Commodity-Logik organisieren wollen und auf öffentliche Unterstützung hoffen können. Gerade in der Tierhaltung gehen Branchenentwicklung und gesellschaftliche Erwartungshaltungen stark auseinander: Der größte tierhaltende Betrieb in Deutschland „produziert“ mehr als 1,5 Mio. Ferkel pro Jahr und damit die Basis für jedes 40. Steak. Verbraucher gehen dagegen - so unsere Befragungsergeb- 
nisse - im Durchschnitt (Median) bei einem Bauernhof mit mehr als 100 Schweinen von Massentierhaltung aus und verbinden damit Enge, Leid und Krankheiten (Kayser et al. 2012b).

Mit Bezug auf Charles Taylor (1995) lässt sich dieses Unbehagen an der modernen Landwirtschaft als Kritik an der Unterwerfung der Natur unter die Verwertungslogik wirtschaftlich-rationalen Handelns interpretieren. Viele Menschen sehen in Natur und Landschaft eine der wenigen übriggebliebenen Ressourcen lebensweltlichen Selbstverständnisses, einen Rückzugsort gegen die Zumutungen der Globalisierung, einen Ort der Identitätsstiftung und Heimat. So aufgeladen wird eine sich von regionalen Bindungen, Naturgrenzen und handwerklichen Traditionen entfernende Branche als Bedrohung erfahren.

Der gesellschaftliche Konflikt um Produktivität versus Natürlichkeit greift dann durch bis auf die Semantik von Werbebegriffen. Bezeichnungen, die eine besondere Natürlichkeit von Lebensmitteln suggerieren, sind bis auf ganz wenige Ausnahmen (z. B. Biolebensmittel) nicht gesetzlich definiert und entsprechend umstritten (Wissenschaftliche Beiräte 2012, Zühlsdorf/Spiller 2013): Bis zu welcher Unternehmensgröße lässt sich von handwerklicher Produktion sprechen, wie lange müssen Kühe auf der Weide stehen, um die Bezeichnung Weidemilch zu rechtfertigen, wie weit dürfen regionale Lebensmittel transportiert werden? Noch basaler geht es um Fragen der Produktidentitäten: Darf in einer Geflügelwurst aus geschmacklichen Gründen Schweinefleisch enthalten sein? Müssen Konsumenten davon ausgehen, dass ein Käsebrötchen mit preiswertem pflanzlichen Fett überbacken wird (Imitatkäse)?

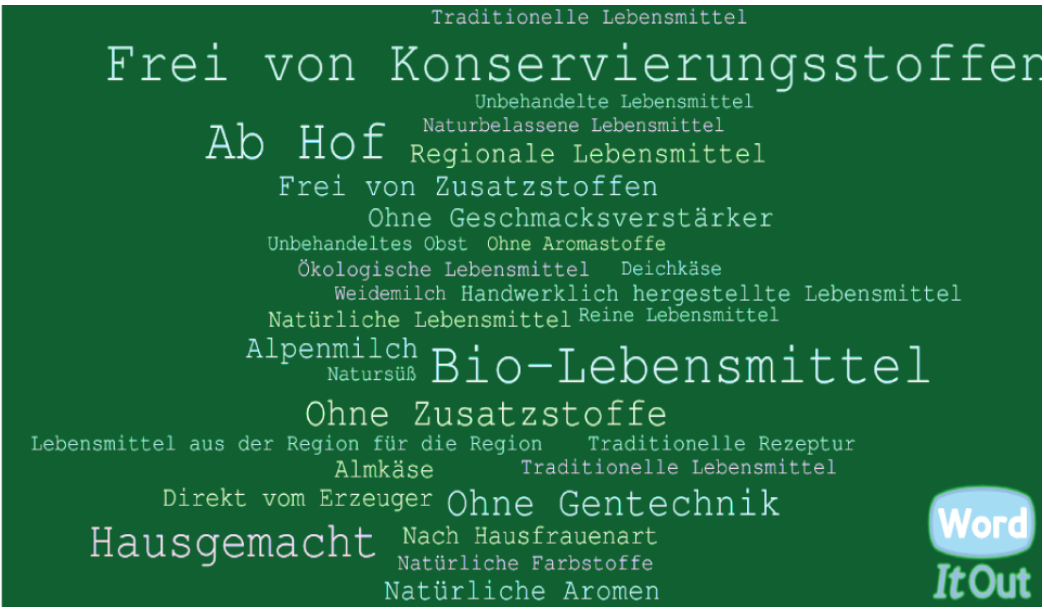

Abb. 2. Marketing-Claims im semantischen Feld „Natürlichkeit“ - Tag Cloud. Quelle: Eigene GoogleTrefferanalyse und Darstellung. 
Natürlichkeitspräferenzen sind damit Teil eines gesellschaftlichen Wertewandels, der weit über die Vermeidung von künstlichen Zusatzstoffen hinausgeht. Marketingstudien zeigen unter dem Terminus „David gegen Goliath“ zunehmend Präferenzen für kleinbetriebliche Anbieter (Sidali et al. 2014). Unternehmen, die ihre uneigennützige Motivation wie z. B. die Liebe zum Produkt betonen, wirken für einen Teil der Verbraucher überzeugender (anti-kapitalistische Attitüde und Underdog-Effekte). Insgesamt geht es um die Intimität von Kundenbeziehungen und wahrgenommene Authentizität (Stockebrand/Spiller 2008).

\section{Fazit}

Deviantes Verhalten wie im Pferdefleischskandal ist zum einen erklärbar durch eine geringe Überwachungsdichte und hohe Gewinnspannen, die Betrug aus ökonomischer Sicht rational erscheinen lassen. In den letzten Jahren wurden Qualitätssicherungssysteme allerdings massiv ausgebaut. Wenn gleichwohl die zeitliche Dichte der Krisenfälle hoch bleibt, so hat dies zwei Ursachen: Zum einen eine Branchenkultur, in der hohes Risiko und kurzfristige Zielsetzungen dominieren, was eine „Spielermentalität“ unter Einschluss illegaler Entscheidungsvarianten begünstigt. Zum anderen wandelt sich der Konflikttyp. Der Pferdefleischskandal steht noch für den Fall klassischer Produktfehler und Produktfälschungen. Diese Krisen werden zunehmend begleitet durch Konflikte entlang der Grenze von Produktivität versus Natürlichkeit, in der sich Prozesse der Marktdifferenzierung einerseits und idealistische Vorstellungen von Natur und Sinnsuche andererseits bündeln.

\section{Literatur}

Abrahamson, E., Fombrun, Ch. J. (1992): Forging the Iron Cage: Interorganizational Networks and the Production of Macro-Culture, in: Journal of Management Studies 29 (2), S. 175-194.

Albersmeier, F., Spiller, A., Jäckel, K. (2008): Öffentlichkeitsorientierung in der Ernährungswirtschaft: Eine empirische Studie zum Umgang mit kritischen Anspruchsgruppen, in: Zeitschrift für Management 3 (4), S. 363-383.

Albersmeier, F., Schulze, H., Jahn, G., Spiller, A. (2009): The reliability of third-party certification in the food chain: From checklists to risk-oriented auditing, in: Food Control 20 (10), S. 927-935.

Böhm, J., Kayser, M., Nowak, B., Spiller, A. (2010): Produktivität vs. Natürlichkeit - Die deutsche Agrar- und Ernährungswirtschaft im Social Web, in: Kayser, M., Böhm, J., Spiller, A. (Hrsg.) (2010): Die Ernährungswirtschaft in der Öffentlichkeit. Social Media als neue Herausforderung der PR, Göttingen: Cuvillier Verlag, S. 105-139.

Deal, T. E., Kennedy, A. A. (1982): Corporate Cultures: The Rites and Rituals of Corporate Life, Harmondsworth.

Europäisches Parlament (2013): Entwurf eines Berichts über die Nahrungsmittelkrise, Betrug in der Nahrungskette und die entsprechende Kontrolle, Ausschuss für Umweltfragen, öffentliche Gesundheit und Lebensmittelsicherheit, http://www.europarl.europa.eu/meetdocs/2009 _2014/documents/envi/pr/1005/1005774/1005774de.pdf. 
Fischler, F. (2012): Mehr Realitätssinn und weniger Agrarromantik, http://www.kreiszeitung.de/ lokales/diepholz/diepholz/mehr-realitaetssinn-weniger-agrarromantik-1613241.html.

Florida, R. (2002): The Rise of the Creative Class. Basic Books, New York.

Franz, A., Deimel, I. und Spiller, A. (2012): Concerns about animal welfare: A cluster analysis of German pig farmers, in: British Food Journal 114 (10), S. 1445-1462.

Hauser, M. (2012): Consumer Value Monitor Food. Wie Konsumenten in Zukunft essen wollen. GDI-Studie Nr. 38, Rüschlikon.

Hofstede, G. (1980): Culture's Consequences: International Differences in Work Related Values, Beverly Hills, CA.

Jahn, G., Schramm, M., Spiller, A. (2005): The Reliability of Certification: Quality Labels as a Consumer Policy Tool, in: Journal of Consumer Policy 28 (1), S. 53-73.

Kayser, M., Böhm, J., Spiller, A. (2012a): Zwischen Markt und Moral - Wie wird die deutsche Landund Ernährungswirtschaft in der Gesellschaft wahrgenommen?, in: Schriften der Gesellschaft für Wirtschafts- und Sozialwissenschaften des Landbaues e. V., Bd. 47, S. 329-341.

Kayser, M., Schlieker, K., Spiller, A. (2012b): Die Wahrnehmung des Begriffs “Massentierhaltung“ aus Sicht der Gesellschaft, in: Berichte über Landwirtschaft 90 (3), S. 417-428.

Lang, T., Heasman, M. (2004): Food Wars: The Global Battle for Mouths, Minds and Markets, Earthscan Publications, 2004.

Padilla Bravo, C., Villanueva Ramirez, I., Neuendorff, J., Spiller, A. (2013): Assessing the impact of unannounced audits on the effectiveness and reliability of organic certification, in: Organic Agriculture, 3 (2), S. 95-109. Doi: 10.1007/s13165-013-0048-9.

Rojas-Méndez, J., Sadrudin, S. A., Claro-Riethmüller, R., Spiller, A. (2012): Acceptance of Genetically Modified Foods with Health Benefits: A Study in Germany, in: Journal of Food Products Marketing 18 (3), S. 200-221.

Schramm, M., Spiller, A., Staack, T. (2004): Brand Orientation in der Ernährungsindustrie, Wiesbaden.

Schulze, H., Böhm, J., Kleinschmit, D., Spiller, A., Nowak, D. (2008a): Öffentliche Wahrnehmung der Primärverantwortung für Lebensmittelsicherheit: Eine Medienanalyse der Gammelfleischskandale, in: Agrarwirtschaft 57 (7), S. 334-345.

Schulze, H., Spiller, A., Böhm, J., de Witte, T. (2008b): Ist Geiz wirklich geil? Preis-Qualitäts-Relationen von Hersteller- und Handelsmarken im Lebensmittelmarkt, in: Agrarwirtschaft 57 (6), S. 299-310.

Schulze, B., Spiller, A. (2008): Trends im Verbraucherverhalten: Ein Forschungsüberblick zum Fleischkonsum, in: Spiller, A., Schulze, B. (Hrsg.): Zukunftsperspektiven der Fleischwirtschaft Verbraucher, Märkte, Geschäftsbeziehungen. Göttingen: Universitätsverlag Göttingen, S. 233-272.

Sidali, K. L., Kastenholz, E., Bianchi, R. (2014): Food tourism, niche markets and products in rural tourism: combining the intimacy model and the experience economy as a rural development strategy, Journal of Sustainable Tourism, http://dx.doi.org/10.1080/09669582.2013.836210.

Spiller, A. (2009): Strategische Spielräume in der deutschen Ernährungsindustrie: Zur Überwindung rigider Branchenkulturen, in: Antoni-Komar, I. et al. (Hrsg.): Neue Konzepte der Ökonomik Unternehmen zwischen Nachhaltigkeit, Kultur und Ethik. Marburg: Metropolis Verlag, S. 349-367.

Stockebrand, N., Spiller, A. (2008): Authentizität als Erfolgsfaktor im Regionalmarketing: Eine erste Skizze, in: Antoni-Komar, I., Pfriem, R., Rabe, T., Spiller, A. (Hrsg.) (2008): Ernährung, Kultur, Lebensqualität. Wege regionaler Nachhaltigkeit. Marburg: Metropolis Verlag, S. 145-166.

Taylor, C. (1995): Das Unbehagen an der Moderne, Suhrkamp, Frankfurt.

Theuvsen, L., Spiller, A., Peupert, T., Jahn, G. (Ed.) (2007): Quality Management in Food Chains, Wageningen. 
Wissenschaftliche Beiräte für Verbraucher- und Ernährungspolitik sowie Agrarpolitik beim Bundesministerium für Ernährung, Landwirtschaft und Verbraucherschutz (2012):

Politikstrategie Food Labelling, in: Berichte über Landwirtschaft 90 (1), S. 35-69.

Zühlsdorf, A., Spiller, A. (2013): Lebensmittelkennzeichnung und -aufmachung aus Sicht der Verbraucher: Deutlicher Nachbesserungsbedarf in punkto Verständlichkeit, in: Deutsche Lebensmittel Rundschau, 109 (11), S. 570-573. 